$\dagger$ Permanent address: Physics Department, Rand Afrikaans University, Johannesburg, South Africa.

${ }^{1}$ R. H. Siemssen, J. V. Maher, A. Weidinger, and

D. A. Bromley, Phys. Rev. Lett. 19, 369 (1967), and 20, 175 (1967); J. V. Maher, M. W. Sachs, R. H. Siemssen, A. Weidinger, and D. A. Bromley, Phys. Rev. 188, 1665 (1969).

${ }^{2}$ R. J. Munn, B. Block, and F. B. Malik, Phys. Rev. Lett. 21, 159 (1968); K. A. Brueckner, J. R. Buchler, and M. M. Kelly, Phys. Rev. 173, 944 (1968).

${ }^{3}$ W. Scheid, R. Ligensa, and W. Greiner, Phys. Rev. Lett. 21, 1479 (1968); W. Scheid and W. Greiner, Z. Phys. 226, 364 (1969).

${ }^{4}$ L. Rickertsen, B. Block, J. W. Clark, and F. B. Malik, Phys. Rev. Lett。22, 951 (1969); R. A. Chatwin,
J. S. Eck, A. Richter, and D. Robson, Phys. Rev。180, 1049 (1969), and Phys. Rev. C 1, 795 (1970).

${ }^{5}$ D. A. Bromley, in Proceedings of the International Conference on Nuclear Reactions Induced by Heavy Ions, Heidelberg, Germany, 15-18 July 1969 (to be published).

${ }^{6}$ B. Block and F. B. Malik, Phys. Rev. Lett. 19 , 239 (1967)。

${ }^{7}$ E. Almqvist, D. A. Bromley, and J. A. Kuehner, Phys. Rev. Lett. $\underline{4}, 515$ (1960)。

${ }^{8}$ B. Imanishi, Phys。Lett. 27B, 267 (1968), and Nucl. Phys. A125, 33 (1969).

${ }^{9} \mathrm{~W}$. Scheid and W. Greiner, to be published.

${ }^{10}$ U. C. Voos, W. v. Oertzen, and R. Bock, Nucl. Phys. A135, 207 (1969).

\title{
ANISOTROPY AND POLARIZATION IN THE GRAVITATIONAL-RADIATION EXPERIMENTS*
}

\section{J. Weber $\uparrow$}

Institute for Advanced Study, Princeton, New Jersey 08540

(Received 8 September 1969; revised manuscript received 24 June 1970)

\begin{abstract}
Large anisotropy is observed for gravitational-radiation-detector intensity as a function of sidereal time, with peaks in the direction of the galactic center and in the opposite direction consistent with $12-\mathrm{h}$ antenna symmetry. The 12-h sidereal-time histograms exhibit anisotropy exceeding 6 standard deviations from the mean.
\end{abstract}

Earlier papers ${ }^{1,2}$ have discussed the conception, analysis, and development of the gravitational-radiation antenna. A coincidence experiment involving detectors at Argonne National Laboratory and the University of Maryland was carried out ${ }^{3.4}$ in 1969 , and gave positive results. Evidence has been presented to support conclusions that the coincidences are not accidental and are not caused by seismic or electromagnetic effects. Results of a study of cosmic-ray interactions, at the Maryland site, have been published. ${ }^{5}$

The antennas are mass quadrupoles and have some directivity. Their axes are horizontal and point east-west at each site. The earth rotates the "antenna beam" among the stars. The time of each coincidence is therefore some measure of the direction of the source. Observation of coincidences over a period of months would be expected to show anisotropy and give information concerning possible sources.

Intensity measurements. - It will be proved that for the detectors (which are harmonic oscillators) the effect of sudden gravitational-radiation excitation is to add the gravitational induced velocity to the initial velocity associated with the thermal fluctuations. The sudden velocity increase decays with the relaxation time of the detector cylinder. Consider a harmonic oscillator excited by a delta function at $t=0$. The equation of motion is

$$
m \ddot{x}+r \dot{x}+k x=A \delta(t) .
$$

The final velocity $\dot{x}_{F}$ just after $t=0$ is given in terms of the initial velocity $\dot{x}_{I}$ just before $t=0$, by integration of (1), as

$$
\dot{x}_{F}=\left(A+m \dot{x}_{I}\right) / m \text {. }
$$

$\dot{x}_{I}$ will vary over a wide range in consequence of the thermal fluctuations. For small signal-tonoise ratio the final velocity $\dot{x}_{F}$ and the energy added in consequence of external excitation will therefore also vary over a considerable range.

A procedure will now be described for measuring, to a good approximation, the square root of the source intensity by observing the detector output, which is mainly noise. Each gravitational-radiation detector employs a chain of linear amplifiers followed by a synchronous square-law detector. All voltages referred to are outputs of the linear amplifiers just before the squarelaw detector. Let $v_{J A}$ be the voltage caused by the $J$ th coincidence if no noise were present, and let $V_{J A}$ be the noise voltage just before excitation, for channel $A$. Let $v_{J B}$ and $V_{J B}$ be the corresponding quantities for channel $B$. Let $\left\langle V_{J_{A}}^{2}\right\rangle$ and $\left\langle V_{J_{B}}^{2}\right\rangle$ be mean-squared values with averages taken only over a $3-\mathrm{sec}$ interval just pre- 
ceding coincidences. Let $\left\langle V_{A}^{2}\right\rangle$ and $\left\langle V_{B}^{2}\right\rangle$ be the mean-squared voltages averaged over all time. The square-law-detector output is continuously recorded and the quantities $\left[V_{J A}+V_{J A}\right]^{2}$ and $\left[V_{J B}\right.$ $\left.+v_{J B}\right]^{2}$ are directly measured for each coincidence. $V_{J A}{ }^{2}$ and $V_{J B}{ }^{2}$ are difficult to measure. Most of the coincidences occur with unequal amplitudes and this implies that $\left\langle v_{J_{A}}{ }^{2}\right\rangle\left\langle\left\langle V_{J_{A}}{ }^{2}\right\rangle\right.$, $\left\langle v_{J_{B}}{ }^{2}\right\rangle\left\langle\left\langle V_{J B}{ }^{2}\right\rangle\right.$. Good estimates for $\left\langle V_{J A}{ }^{2}\right\rangle$ and $\left\langle V_{J B}{ }^{2}\right\rangle$ are therefore obtainable from the threshold voltages required to trigger coincidences. The normalized intensity for the $J$ th coincidence $I_{J}$ is taken to be

$$
\begin{aligned}
I_{J}=\frac{\left[V_{J A}+V_{J A}\right]^{2}-\left\langle V_{J A}{ }^{2}\right\rangle}{\left\langle V_{A}{ }^{2}\right\rangle} & \\
& +\frac{\left[V_{J B}+v_{J B}\right]^{2}-\left\langle V_{J B}{ }^{2}\right\rangle}{\left\langle V_{B}{ }^{2}\right\rangle} .
\end{aligned}
$$

An on-line computer records coincidences if both channels were initially below threshold and both suddenly increased. Therefore $v_{J A}, v_{J B}$, $V_{J A}$, and $V_{J B}$, always have the same sign. Expression (3) is measured in the following way: Each two-detector coincidence is characterized by two numbers $N_{A}$ and $N_{B} . N_{A}$ is the number of times per day that the amplitude at coincidence in channel $A$ is equalled or exceeded on the average, with a corresponding meaning for $N_{B}$ in channel $B$.

The output-voltage detectors in use are linear for small voltages and approximately square law in the range from twice the mean-square to seven times the mean-square voltage. For the square-law region, $N_{A}$ and $N_{B}$ are given by the empirical approximations

$$
\begin{aligned}
& N_{A}=K \exp \left\{-\alpha\left[V_{J_{A}}+v_{J_{A}}\right]^{2} /\left\langle V_{A}{ }^{2}\right\rangle\right\}, \\
& N_{B}=K \exp \left\{-\alpha\left[V_{J_{B}}+v_{J_{B}}\right]^{2} /\left\langle V_{B}{ }^{2}\right\rangle\right\},
\end{aligned}
$$

where $\alpha$ is measured as 1.64 and $K$ is measured as 10120 , to a precision of about $10 \%$. These expressions describe the present experiment, but are not valid for true square-law detectors.

The normalized intensity for each coincidence is taken to be

$$
\begin{gathered}
\frac{\left[V_{J A}+V_{J A}\right]^{2}-\left\langle V_{J A}{ }^{2}\right\rangle}{\left\langle V_{A}{ }^{2}\right\rangle}+\frac{\left[V_{J B}+V_{J B}\right]^{2}-\left\langle V_{J B}{ }^{2}\right\rangle}{\left\langle V_{B}{ }^{2}\right\rangle} \\
=\frac{2 \ln K-\ln N_{A} N_{B}}{\alpha}-\frac{\left\langle V_{J A}{ }^{2}\right\rangle}{\left\langle V_{A}{ }^{2}\right\rangle}-\frac{\left\langle V_{J B}{ }^{2}\right\rangle}{\left\langle V_{B}{ }^{2}\right\rangle} .
\end{gathered}
$$

The right-hand side of Expression (4) is then determined from measurements of $N_{A}$ and $N_{B}$. This procedure was employed because only penand-ink records were available. The gain and zero shifts varied during the seven months of observations. Better precision is obtained than by direct measurements of the left-hand side of (4) even though $N_{A}$ and $N_{B}$ are not precisely known, and for values smaller than $25 / d$, involve errors as large as 50\%. $\left\langle V_{J_{A}}{ }^{2}\right\rangle /\left\langle V_{A}{ }^{2}\right\rangle$ and $\left\langle V_{J_{B}}{ }^{2}\right\rangle /$ $\left\langle V_{B}^{2}\right\rangle$ are $\sim 2.7$.

The summation of the right-hand side of (4) for a given period of solar or sidereal time is shown on the histograms, and the summation of the lefthand side becomes

$$
I=\sum_{J} \frac{2 v_{J A} V_{J A}+v_{J A}^{2}}{\left\langle V_{A}^{2}\right\rangle}+\frac{2 v_{J B} V_{J B}+v_{J B}^{2}}{\left\langle V_{B}^{2}\right\rangle} .
$$

Expression (5) is the detector intensity, which is nearly proportional to the square root of the appropriate mean source intensity.

Figure 1(a) is a histogram of detector intensity versus sidereal time on a $24-\mathrm{h}$ basis, and verifies the 12-h symmetry expected of the antennas. Figure 1(b) is a histogram of detector intensity versus sidereal time on a 12-h basis, with 12 to $24 \mathrm{~h}$ displayed over the 0 - to 12 -h parts. Sidereal time at a point halfway between Argonne National Laboratory and the University of Maryland is employed. Figures 2(a) and 2(b) are histograms of detector intensity versus daylight-saving time at the Maryland site, on 24- and 12-h bases, respectively.

Fluctuations are associated with statistics of the source and statistics of the detectors. For the source, Poisson statistics are assumed. Three-detector-coincidence rates suggest that less than $10 \%$ of the events are being observed. The fluctuations associated with the detectors dominate and the standard deviations shown are computed from the coincidence data. A check on the standard deviations displayed on the histograms can be obtained in the following way. The sidereal-time histograms are the only ones which clearly show anisotropy. Since the observation time is roughly 6 months, the coincidences in each 4-h section of sidereal time will be uniformly distributed over $12 \mathrm{~h}$ of solar time, except for effects of sensitivity fluctuations for extended periods. The anisotropy should very nearly average out in solar time. The histograms of Fig. 2 are expected to represent horizontal straight lines and the six-block histogram permits an in- 

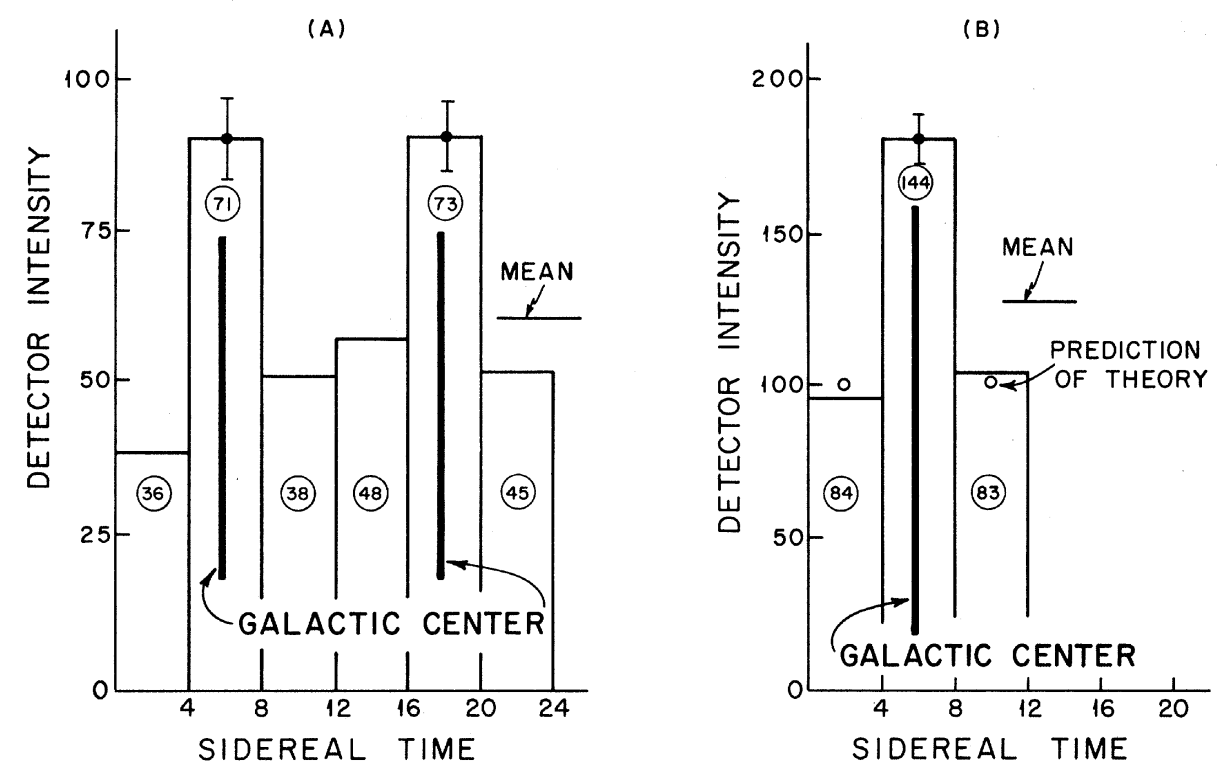

FIG. 1. (a) 24-h histogram of detector intensity versus sidereal time. (b) 12-h histogram of detector intensity versus sidereal time. The encircled numbers are the numbers of coincidences in each bin.

dependent calculation of the standard deviation. The latter would be expected to exceed the value calculated from the assemblage of data because of incomplete averaging out of the anisotropy, and is, in fact, $12 \%$ larger.

A total of 311 coincidences are included, extending down to energies roughly a few per cent of the mean noise energy. About $\frac{1}{10}$ of the intensity and $\frac{1}{5}$ of the number of coincidences are ascribed to accidentals. The period of observation is from 12 May to 14 December 1969.
Theory of the antenna directivity. - The antenna measures the Fourier transform of one component of the Riemann curvature tensor. The quantity measured is really the scalar $R_{\mu \alpha \beta \gamma} A^{\mu} B^{\alpha}$ $\times A^{\beta} B^{\gamma}$. $A^{\mu}$ and $B^{\alpha}$ are vectors depending on details of the experiment and the coordinate system. Let us choose normal coordinates with the axis of the cylinder in direction $x^{1} . A^{\mu}$ has components (zero to three) given by $0,1,0$, and 0 , and $B^{\alpha}$ has components $1,0,0$, and 0 . The appropriate component which drives the detector is
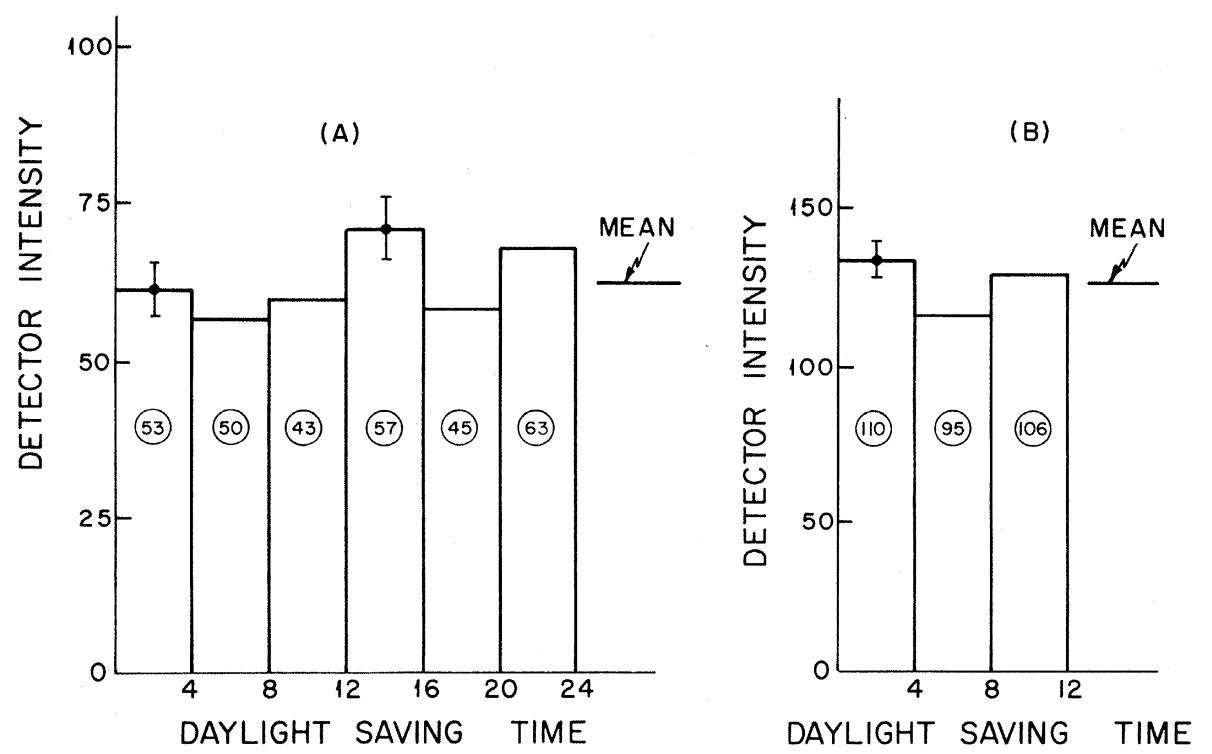

FIG. 2. (a) 24-h histogram of detector intensity versus daylight-saving time at Maryland. (b) 12-h histogram of detector intensity versus daylight-saving time at Maryland. The encircled numbers are the numbers of coincidence in each bin. 
$R_{1010}$. To obtain the directivity pattern, consider the source in a rotated coordinate system labeled by $x^{\prime} y^{\prime} z^{\prime}$ as shown in Fig. 3. One polarization can be represented by the components $R_{1010}$ and $R_{2020}{ }^{\prime}$. The transformation properties of the Riemann tensor then lead to

$$
R_{1010}=R_{1010}^{\prime} \frac{\partial x^{\prime 1}}{\partial x^{1}} \frac{\partial x^{\prime 1}}{\partial x^{1}}+R_{2020}^{\prime} \frac{\partial x^{\prime 2}}{\partial x^{1}} \frac{\partial x^{\prime 2}}{\partial x^{1}}
$$

Einstein's field equations for vacuum require that

$$
R_{\mu \nu}{ }^{\prime}=R_{1010}{ }^{\prime}+R_{2020}{ }^{\prime}=0 .
$$

The partial derivatives in (6) may be evaluated from the direction cosines and give the result

$$
R_{1010}=R_{1010}{ }^{\prime}\left[\left(1+\cos ^{2} \theta\right) \cos ^{2} \varphi-1\right] .
$$

(8) is a maximum for $\varphi=\frac{1}{2} \pi, \frac{3}{2} \pi$, corresponding to a source on the meridian for $x^{1}$ pointing east and west. The second independent state of polarization may be described by the Riemann-tensor components $R_{1020}{ }^{\prime}$ and $R_{2010}{ }^{\prime}$. Analysis similar to that leading to (8) then gives

$$
R_{1010}=-R_{1020}{ }^{\prime} \cos \theta \sin 2 \varphi \text {. }
$$

(9) is zero for a source on the meridian and is a maximum for $\varphi= \pm \frac{1}{4} \pi, \pm \frac{3}{4} \pi$.

These expressions for the directivity, associated with two independent states of polarization, are exact. It is important that only Einstein's field equations and the transformation properties of a tensor under rotations are needed to derive them. Similarly, the theory of the measurement of the Riemann tensor by these antennas involves no approximation other than neglect of the gravitational field of the antenna itself.

For a mixed-polarization source, a suitable squared combination of (8) and (9) is required. There are eight extrema with values dependent on the polarization mixture. There can be two equal large peaks, two equal small peaks, and four equal minima. Another possibility is two deep minima, two equal shallow minima, and four equal large peaks.

An unpolarized source would be expected to have the average intensity function

$$
\begin{aligned}
f(\theta, \varphi)=\left[\left(1+\cos ^{2} \theta\right) \cos ^{2} \varphi-1\right]^{2} & \\
& +\cos ^{2} \theta \sin ^{2} 2 \varphi
\end{aligned}
$$

Discussion of experimental results. - Figures 1(a) and 1(b) suggest that the Argonne-Maryland gravitational-radiation-detector array is responding to radiation from the direction of the

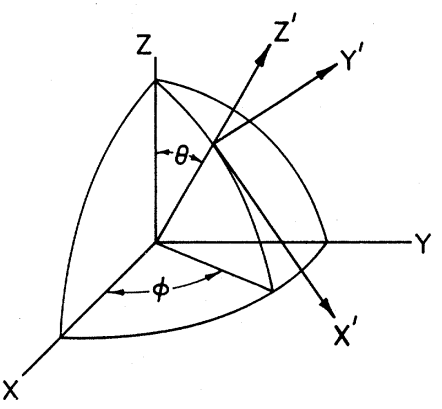

FIG. 3. Coordinates for calculation of antenna directivity.

galactic center. The peak at $4-8 \mathrm{~h}$ of Fig. 1(a) corresponds to the antenna viewing the galatic center through the earth. This suggests that gravitational radiation passes through the earth without significant attenuation.

Let us assume that the galactic center is an unpolarized source and employ (5) and (10) to calculate the expected ratio of the center to the adjacent histogram blocks of Fig. 1(b). The 15deg departure from parallelism due to the difference in longitudes of the Argonne and Maryland sites was taken into account, and corrections were applied for the accidentals. The predicted height of the 0 - to 4- and 8- to 12-h blocks is shown on Fig. 1(b), normalized to unity for the center peak. Agreement of theory and experiment is seen to be good.

The galactic center has both attractive and unattractive features. A good feature is the fact that $10^{10}$ solar masses are there and it is reasonable to find the source to be the region of the sky containing most of the mass of the galaxy. The galactic center is far away -8000 pc. If we assume the validity of existing theory, that the radiation is isotropic and that there are no focusing effects, what is being observed may only amount to roughly $10^{-2}$ solar masses per year. This is a relatively small energy loss which can be converted to a very large one if certain further assumptions are made. The narrow detector bandwidth, $\sim 0.03 \mathrm{~Hz}$, suggests a flux of roughly 0.3 solar masses per $\mathrm{Hz}$ bandwidth. We may guess that perhaps we are only seeing $10 \%$ of what is emitted and that the power spectrum extends over several hundred $\mathrm{Hz}$. Numbers like 1000 solar masses per year then come into view. Lack of isotropy and focusing effects might very substantially reduce this. Only the theory of the directivity and the measurement of the Riemann tensor are exact. Expressions for the antenna cross section require very reasonable assump- 
tions and approximations still untested by experiment. It is also possible that the antenna is operating in a more sensitive mode than ordinarily assumed. Frozen-in metastable configurations within each detector might decay to equilibrium as a result of collective excitation by gravitational radiation, releasing far more energy than implied by the gravitational-radiation flux.

I am also studying the possibility that cosmological gravitational radiation is being observed, focused by the galactic center.

Conclusion. - The large (exceeding 6 standard deviations) sidereal anisotropy is evidence that the gravitational-radiation-detector coincidences are due to a source or sources outside the solar system. The location of the peaks suggests that the source is the $10^{10}$ solar masses at the galactic center.

I have received very helpful suggestions for doing these experiments and processing the data from L. W. Alvarez, F. Crawford, R. Glasser,
R. H. Dicke, and F. J. Dyson. Enlightening discussions with my chairman, Professor Howard J. Laster, have acquainted me with the past difficulties of cosmic-ray investigations of anisotropy. I thank D. J. Gretz and J. Peregrin for their skill and devotion in maintenance and operation of the Argonne-Maryland antenna array.

\footnotetext{
*Work supported in part by the National Science Foundation.

$\dagger$ On leave from University of Maryland, College Park, Md. 20742.

${ }^{1} \mathrm{~J}$. Weber, Phys. Rev. 117, 306 (1960), and see also General Relativity and Gravitational Waves (Interscience, New York, 1962), Chap.8.

${ }^{2} \mathrm{~J}$. Weber, Relativity Groups and Topology (Gordon and Breach, New York, 1964), p. 875, and Phys. Rev. Lett. 17, 1228 (1966)。

${ }^{3}$ J. Weber, Phys. Rev. Lett。22, 1320 (1969).

${ }^{4} \mathrm{~J}$. Weber, Phys. Rev. Lett. 24, 276 (1970).

${ }^{5}$ D. H. Ezrow, N. S. Wall, J. Weber, and G. B. Yodh, Phys.Rev。Lett. 24, 945 (1970).
}

\title{
POSSIBLE EXOTIC EXCHANGE IN $p n \rightarrow \Delta^{-{ }^{-} \Delta^{++}}$at $6.98 \mathrm{GeV} / c$
}

G. Yekutieli, D. Yaffe, S. Toaff, A. Shapira, E. E. Ronat, U. Karshon, B. Haber, and Y. Eisenberg Department of Nuclear Physics, Weizmann Institute of Science, Rehovot, Israel (Received 15 April 1970)

\begin{abstract}
The reaction $p n \rightarrow \Delta^{-} \Delta^{++}$, where $\Delta^{-}$is emitted in the forward direction in the c.m. system, is observed at $6.98 \mathrm{GeV} / c$ with a cross section of $0.09 \pm 0.03 \mathrm{mb}$. The ratio between this reaction and the reaction $p n \rightarrow \Delta^{++} \Delta^{-}$with $\Delta^{++}$in the forward direction in the c.m. system is found to be $\sigma\left(\Delta^{++} \Delta^{-}\right) / \sigma\left(\Delta^{-} \Delta^{++}\right)=12.2 \pm 4.5$. The $\Delta^{-} \Delta^{++}$production is discussed in terms of exotic meson exchange and double meson exchange.
\end{abstract}

In this Letter we report on the reaction

$$
p n \rightarrow \Delta^{-}(1236) \Delta^{++}(1236)
$$

at $6.98 \mathrm{GeV} / c$, where the $\Delta^{-}$is emitted in the forward direction in the c.m. system with respect to the incident proton. Reaction (1) cannot be described by the exchange of any known meson in the $t$ channel. In this sense (1) is considered a forbidden reaction, and it is expected to have, at higher energy, much smaller cross section than the allowed reaction

$$
p n \rightarrow \Delta^{++} \Delta^{-} \text {, }
$$

with the $\Delta_{.^{++}}$emitted in the forward direction in the c.m. system.

The peripheral description of Reaction (1) requires either (a) the exchange of a doubly charged exotic meson in the $t$ channel or (b) the successive exchange of two charged mesons (see Fig. 1). Both Reactions (1) and (2) were observed at 3.7
$\mathrm{GeV} / c$ by Cohn et al. ${ }^{1}$ The 94000 pictures of the $p d$ experiment at
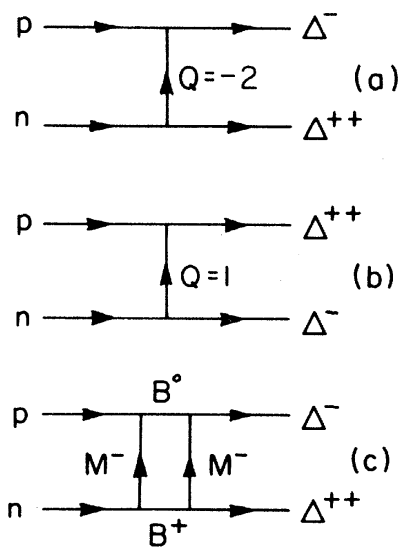

FIG. 1. Exchange diagrams for the reactions (a) $p n$ $\rightarrow \Delta^{-} \Delta^{++}$with a single $Q=-2$ exchange, (b) $p n \rightarrow \Delta^{++} \Delta^{-}$ with a single $Q=1$ exchange, and (c) $p n \rightarrow \Delta^{-} \Delta^{++}$with the exchange of two negative mesons. 